\title{
Ancrage du conseil éthique dans le quotidien clinique
}

\section{Jean Martin}

Dr med., membre de la rédaction et ancien membre de la Commission nationale d'éthique

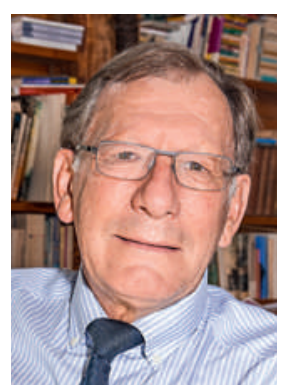

L’Académie suisse des sciences médicales a émis en 2012 des recommandations sur le soutien éthique en médecine. Elle a organisé le 21 avril dernier à Berne un symposium auquel ont participé une centaine de professionnels, faisant le point sur les enjeux et les perspectives d'avenir. D'abord, il convient de relever que (comme toujours, pourrait-on dire) il n'y a pas une manière standard, qui serait la seule bonne, d'implanter le conseil éthique dans un hôpital, un EMS ou la pratique ambulatoire. La chargée d'éthique de la clinique St. Anna de Lucerne a dit que «l'essentiel n'est pas comment une situation difficile est analysée, mais qu'elle le soit». La formule pourra surprendre, mais le fait est que des chemins différents sont possibles. La première condition est que les responsables organisationnels/exécutifs accordent à cette fonction la place nécessaire - et des moyens qui permettent de l'assumer. Un orateur a relevé qu'un scandale peut être le facteur déclenchant d'une préoccupation éthique institutionnelle, mais il est évidemment préférable de ne pas attendre une telle occurrence!

La possible professionnalisation du conseil éthique a été évoquée. Chez nous et à court terme, il ne paraît pas que la solution puisse être recherchée dans une exigence de formation unique aux conditions rigides. En effet, la fonction est assumée par des personnes dont la formation de base peut être aussi bien la médecine, une profession soignante, la philosophie ou la psychologie, voire le droit. Cela étant, elles doivent avoir bénéficié d'une expérience pertinente, aussi au plan théorique, et démontré leur engagement dans ce domaine - y compris un talent pour la réflexion et le débat interdisciplinaires.

Le prof. Bert Molewijk, d'Amsterdam, a présenté la «moral case deliberation», approche contextuelle et pragmatique de l'éthique clinique, ancrée dans l'expérience des professionnels qui apportent leurs interrogations dans un dialogue structuré, facilité par un éthicien [1]. «Le soutien éthique clinique n'est pas une fin en soi», a-t-il dit, allusion à ce qu'il s'agit d'un rôle/ prestation de service aux praticiens qui sont au front. Il a souligné l'importance de stimuler, chez ceux qui sollicitent une appréciation, un sentiment de co-propriété et de co-responsabilité dans la démarche éthique et les conclusions qu'on en tire. Rappelons que les commissions ou consultants en éthique sont là pour délibérer des valeurs, droits et intérêts en jeu dans la situation soumise, et pour esquisser les avenues/actions possibles (cas échéant la «moins mauvaise» avenue). Notamment aussi pour discuter de désaccords apparus dans l'équipe. Ce faisant, ils formulent des analyses ou recommandations mais, en règle générale, la responsabilité des décisions pratiques, au lit du malade, reste avec le médecin et l'équipe soignante. En bref: on ne saurait imposer des positions éthiques, on en débat et on s'efforce d'arriver à un consensus.

La responsable de l'unité d'éthique d'un hôpital universitaire a noté que, le plus souvent, ce sont les chefs de service qui soumettent une situation complexe plutôt que des collègues juniors. Y aurait-il chez ces derniers la crainte qu'une demande de leur part soit vue comme un signe d'insuffisance? Alors que, bien souvent, dire «Je ne sais pas» doit être vu comme une forme de responsabilité, de courage même. Et il faut plus de courage encore pour attirer l'attention d'un chef sur des aspects éthiquement discutables de la pratique dans son service... Une personne œuvrant dans un cadre déontologique plutôt traditionnel a relevé que les désaccords entre les médecins et un corps infirmier plus ouvert étaient fréquents. Ellen Fox, spécialiste américaine de l'Université Clarkson, a parlé de "psychological safety»: pour des débats fructueux, la sécurité psychologique, en d'autres termes la confiance au sein de l'équipe est un facteur majeur, nourri par une atmosphère d'écoute et de respect mutuel.

L'objectif est l'émergence d'une véritable culture éthique partagée dans les prestations de soins (à ce dernier sujet, noter la parution récente d'un utile ouvrage romand [2]). A été rappelée aussi, comme un guide simple, la formule de l'éthicien genevois Eric Fuchs disant que le but de l'éthique, c'est chercher «comment faire pour faire bien».

\section{Références}

1 Molewijk B, Abma T, Stolper M, Widdershoven G. Teaching ethics in the clinic. The theory and practice of moral case deliberation. Journal Med. Ethics. 2008;34:120-4.

2 Corbaz $\mathrm{P}$, Quinche $\mathrm{F}$. Ethiques pour les soins à domicile. Genève: Editions Médecine et Hygiène; 2015. 